\title{
Synthesis of New Pyrazole Derivatives Containing 2-Methylquinoline Ring System: A Novel Class of Potential Antimicrobial Agents
}

\author{
G. BHEEMA RAJU, M. MAHESH, G. MANJUNATH and P. VENKATA RAMANA* \\ Department of Chemistry, Sri Krishnadevaraya University, \\ Ananthapuramu - 515 003, Andhra Pradesh, India \\ ramanapv54@gmail.com
}

Received 26 October 2015 / Accepted 20 November 2015

\begin{abstract}
Two series of diversely substituted 1-(3,5-dimethyl-4-(substitutedphenyldiazenyl)-1Hpyrazol-1-yl)-2-((2-methylquinolin-8-yl)oxy)ethanones (8a-g) and 3-methyl-1-(2-((2-methylquinolin-8-yl)oxy)acetyl)-4-(2-substitutedphenylhydrazono)-1 $H$-pyrazol-5(4H)-ones (9a-h) were synthesized. All the newly synthesized compounds were characterized by IR, ${ }^{1} \mathrm{H}$ NMR, ${ }^{13} \mathrm{C}$ NMR, Mass and chemical analysis. The compounds were evaluated for their in vitro antibacterial activity against some Gram-positive bacteria, Staphylococcus aureus, Bacillus subtilis, Gram-negative bacteria, Escherichia coli, Pseudomonas aeruginosa, Salmonella typhi and screened for antifungal activity against Aspergillus niger, Ustilago maydis using the agar disc diffusion method. All the tested compounds showed significant antibacterial activity against both the strains with compounds 8d and 9d being more potent.
\end{abstract}

Keywords: 2-Methylquinolin-8-ol, 2-((2-Methylquinolin-8-yl)oxy) acetohydrazide, Azopyrazoles, hydrazonopyrazolin-5-ones, Antibacterial and antifungal activities

\section{Introduction}

Heterocyclic compounds played a vital role in biological processes and are wide spread as natural products. They are widely found in nature particularly in nucleic acids, plant alkaloids, anthocyanins and flavones as well as in haem and chlorophyll. Additionally some vitamins, proteins, hormones contain aromatic heterocyclic system. Synthetically produced heterocycles designed by organic chemists are used for instance as agrochemicals and pharmaceuticals and play an important role in human life. Heterocycles have enormous potential as the most promising molecules as lead structures for the design of new drugs ${ }^{1-4}$.

Pyrazoles have a unique place and contributed significantly to biological, medicine, pharmacology fields. Pyrazole ring containing substituted five membered ring exhibited antibacterial, antifungal, tumor-necrosis inhibitor, antimicrobial, hypoglycemic, hypolipidemic and anti-inflammatory activity ${ }^{5-15}$. On the other hand quinoline and their 
derivatives could be considered as possible antimalarial, anti-bacterial, antifungal, anthelmintic, cardiovascular, anticonvulsant, anti-inflammatory and analgesic activity ${ }^{16-21}$. Hence it was thought of interest to accommodate pyrazole and quinoline moieties in a singular molecular frame work to synthesize the linked heterocyclics for enhancing biological activity. Two different series of quinoline ring substituted pyrazole derivatives (8a-g and 9a-h) were synthesised and screened for their antibacterial activity.

\section{Experimental}

Melting points were determined in open capillaries on a Mel-Temp apparatus and are uncorrected. The homogeneity of the compounds was checked by TLC (silica gel H, BDH, ethyl acetate-hexane, 3:5). The IR spectra were recorded on Perkin Elmer 100 FT-IR spectrometer as $\mathrm{KBr}$ pellets. The wave numbers were given in $\mathrm{cm}^{-1}$. The ${ }^{1} \mathrm{H}$ NMR spectra were recorded in $\mathrm{CDCl}_{3} / \mathrm{DMSO}-\mathrm{d}_{6}$ on a jeol JNM $\lambda-400 \mathrm{MHz}$ machine. The ${ }^{13} \mathrm{C}$ NMR spectra were recorded in $\mathrm{CDCl}_{3} / \mathrm{DMSO}_{-} \mathrm{d}_{6}$ on a Jeol JNM spectrometer operating at $100 \mathrm{MHz}$. All chemical shifts are reported in $\delta(\mathrm{ppm})$ using TMS as an internal standard. The mass spectra were recorded on VG $7070 \mathrm{H}$ mass spectrometer. The microanalyses were performed on Perkin-Elmer 240C elemental analyzer. All newly synthesized compounds yielded spectral data consistent with the proposed structure and microanalysis data are in agreement with the theoretical values.

Synthesis of ethyl 2-((2-methylquinolin-8-yl)oxy)acetate (2)

A mixture of 2-methylquinolin-8-ol (1) 0.01 mole, ethyl chloroacetate (0.01 mole) and anhydrous $\mathrm{K}_{2} \mathrm{CO}_{3}(1.38 \mathrm{~g}, 0.01$ mole and $10 \mathrm{~mL}$ of DMF) was subjected to agitation at room temperature for $8 \mathrm{~h}$. The reaction mixture was diluted with ice cold water and the separated product was filtered, washed with water and recrystallized from ethanol. $80 \%$ yield, $\mathrm{mp} 55^{\circ}-57^{\circ} \mathrm{C}$.

IR $\left(\mathrm{cm}^{-1}\right)$ : $3065\left(\mathrm{CH}\right.$ stretching in aromatics), $2930\left(\mathrm{CH}\right.$ stretching in $\left.\mathrm{CH}_{3} / \mathrm{CH}_{2}\right), 1730$ $\left(\mathrm{C}=\mathrm{O}\right.$ stretching in acetates), $1220,1010\left(\mathrm{sp}^{2} / \mathrm{sp}^{3} \mathrm{C}-\mathrm{O}\right.$ stretching); ${ }^{1} \mathrm{H}$ NMR (DMSO-d 6 , 300 $\mathrm{MHz}): \delta 1.22\left(\mathrm{t}, 3 \mathrm{H}, \mathrm{CH}_{3}\right.$ ethyl), $2.50\left(\mathrm{~s}, 3 \mathrm{H}, \mathrm{CH}_{3}\right.$ quinoline ring), 4.20 (q, $2 \mathrm{H}, \mathrm{CH}_{2}$ ethyl), 5.04 $\left(\mathrm{d}, 2 \mathrm{H}, \mathrm{OCH}_{2}\right), 7.13$ (d, 1H,H3 quinoline ring), 8.34 (d, 1H,H4 quinoline ring), 7.57 (d, $1 \mathrm{H}, \mathrm{H} 5$ quinoline ring), 7.55 (t, $1 \mathrm{H}, \mathrm{H} 6$ quinoline ring), 7.47 (d, $1 \mathrm{H}, \mathrm{H} 7$ quinoline ring); ${ }^{13} \mathrm{C}$ NMR (DMSO-d $6,300 \mathrm{MHz}): \delta 13.8\left(\mathrm{CH}_{3}\right.$ ethyl), $20.8\left(\mathrm{CH}_{3}\right.$ quinoline ring), $63.1\left(\mathrm{CH}_{2}\right.$ ethyl), 153.5, 122.4, 136.3, 121.3, 124.2, 112.4, 155.3, 139.3, 129.0 (quinoline ring), 67.1 $\left(\mathrm{OCH}_{2}\right), 165.7$ (CO acetate); Anal. Calcd for $\mathrm{C}_{14} \mathrm{H}_{15} \mathrm{NO}_{3}: \mathrm{C}, 68.56 ; \mathrm{H}, 6.16 ; \mathrm{N}, 5.71$. Found: $\mathrm{C}, 68.22 ; \mathrm{H}, 5.94 ; \mathrm{N}, 5.62 \%$.

\section{Synthesis of 2-((2-methylquinolin-8-yl)oxy)acetohydrazide (3)}

To a solution of ethyl 2-((2-methylquinolin-8-yl)oxy)acetate (2) (0.01 mole) in methanol, hydrazine hydrate $(0.02$ mole $)$ was added and the reaction mixture was refluxed for $8 \mathrm{~h}$. The excess of the solvent was distilled off and the reaction mixture was cooled. The separated solid was filtered, washed with pet. ether $\left(40^{\circ}-60^{\circ} \mathrm{C}\right)$ and recrystallized from water.

Yield 70\%; m.p: $125^{\circ}-127^{\circ} \mathrm{C}$; IR $\left(\mathrm{cm}^{-1}\right)$ : 3325 (NH stretching), $3070(\mathrm{CH}$ stretching in aromatics), 2910 ( $\mathrm{CH}$ stretching in $\left.\mathrm{CH}_{3} / \mathrm{CH}_{2}\right), 1650$ (C=O amide), 1260, $1040\left(\mathrm{sp}^{2} / \mathrm{sp}^{3} \mathrm{C}-\mathrm{O}\right.$ stretching); ${ }^{1} \mathrm{H}$ NMR (DMSO-d 6 , $300 \mathrm{MHz}$ ): $\delta 2.50$ (s, 3H, $\left.\mathrm{CH}_{3}\right), 4.44$ (s, 2H, $\mathrm{NH}_{2}$ ), 4.75 (s, $\left.2 \mathrm{H}, \mathrm{OCH}_{2}\right), 7.24(\mathrm{~d}, 1 \mathrm{H}, \mathrm{H} 3$ quinoline ring), $8.36(\mathrm{~d}, 1 \mathrm{H}, \mathrm{H} 4$ quinoline ring $), 7.58(\mathrm{t}, 1 \mathrm{H}, \mathrm{H} 5$ quinoline ring), $7.56(\mathrm{~d}, 1 \mathrm{H}, \mathrm{H} 6$ quinoline ring), 7.51(d,1H,H7 quinoline ring), $9.47(\mathrm{~s}, 1 \mathrm{H}$, $\mathrm{NH}) ;{ }^{13} \mathrm{C}$ NMR (DMSO-d $\left.6,100 \mathrm{MHz}\right): \delta 20.2\left(\mathrm{CH}_{3}\right), 153.4,122.5,135.8,121.4,123.8$, 112.4, 154.8, 139.5, 130.1 (quinoline ring), $67.5\left(\mathrm{OCH}_{2}\right), 162.8$ (CO amide); Anal. Calcd for $\mathrm{C}_{12} \mathrm{H}_{13} \mathrm{~N}_{3} \mathrm{O}_{2}: \mathrm{C}, 62.33 ; \mathrm{H}, 5.67 ; \mathrm{N}, 18.17$. Found: $\mathrm{C}, 62.16 ; \mathrm{H}, 5.52 ; \mathrm{N}, 18.05 \%$. 


\section{General procedure for the synthesis of 1-chloro-2-substitutedphenyldiazene (5a-h)}

The required primary amine (4a-h) was dissolved in a suitable volume of water containing 2.5-3.5 equivalents of hydrochloride acid or (sulphuric acid) by the application of heat if necessary ${ }^{22}$. The solution thus obtained was cooled to $0{ }^{\circ} \mathrm{C}$ where the amine hydrochloride or (sulphate) usually crystallizes. The temperature was maintained at $0^{\circ}$ to $5{ }^{\circ} \mathrm{C}$ and the aqueous sodium nitrite solution was added portion wise till there was free nitrous acid. The solution was tested for the later with an external indicator paper (moist potassium iodidestarch paper). An excess of acid was maintained to stabilize the diazonium salt solution. However, in those cases where a large excess of acid was harmful, the concentration of the acid was reduced to optimum value.

\section{General procedure for the synthesis of compounds (6a-g)}

A solution of sodium acetate $(100 \mathrm{~g})$ in $100 \mathrm{~mL}$ aqueous alcohol $(50 \%)$ was added to a solution of acetylacetone $(100 \mathrm{~g})$ in $500 \mathrm{~mL}$ of ethanol and the mixture was cooled. To this cold mixture, the corresponding diazonium chloride (5) was added gradually till turbidity was observed ${ }^{6}$. The addition was continued till yellow crystals separated out. These crystals were filtered washed with water and recrystallized from ethanol. Compounds 6a-g are synthesized on the same lines. Yield 70-85\%; m.p: 6a, $92^{\circ} \mathrm{C} ; \mathbf{6 b}, 98^{\circ} \mathrm{C} ; \mathbf{6 c}, 139-140^{\circ} \mathrm{C} ; \mathbf{6 d}$, $233^{\circ} \mathrm{C} ; \mathbf{6 e}, 112^{\circ} \mathrm{C} ; \mathbf{6 f}, 120^{\circ} \mathrm{C} ; \mathbf{6 g}, 120-122^{\circ} \mathrm{C}$.

\section{General procedure for the synthesis of compounds (7a-h)}

A solution of sodium acetate $(100 \mathrm{~g})$ in $100 \mathrm{~mL}$ aqueous alcohol $(50 \%)$ was added to a solution of ethyl acetoacetate $(100 \mathrm{~g})$ in $500 \mathrm{~mL}$ of ethanol and the mixture was cooled. To this cold mixture, the corresponding diazonium chloride (5) was added gradually till turbidity was observed ${ }^{6}$. The addition was continued till yellow crystals separated out. These crystals were filtered washed with water and recrystallized from ethanol. Compounds 7a-h are synthesized on the same lines. Yield 70-85\%; m.p: 7a, $184^{\circ} \mathrm{C} ; \mathbf{7 b}, 178-179^{\circ} \mathrm{C} ; \mathbf{7 c}$, $116-118^{\circ} \mathrm{C} ; \mathbf{7 d}, 195-196^{\circ} \mathrm{C} ; \mathbf{7 e}, 119-120^{\circ} \mathrm{C} ; \mathbf{7 f}, 125-126^{\circ} \mathrm{C} ; \mathbf{7 g}, 90-91^{\circ} \mathrm{C} ; \mathbf{7 h}, 89-91{ }^{\circ} \mathrm{C}$.

General procedure for the synthesis of compounds (8a-g)

A mixture of 3-(phenyldiazenyl) pentane-2,4-dione (6a-g) $(0.01 \mathrm{~mol})$ and 2-((2methylquinolin-8-yl)oxy)acetohydrazide (3) $0.01 \mathrm{~mol}$ in ethanol $(20 \mathrm{~mL})$ was heated under reflux for $8 \mathrm{~h}$. on a water bath. After completion of the reaction, ethanol was evaporated; the residue was dissolved in water, neutralized with $\mathrm{NaHCO}_{3}$ and extracted with ether. The ether solution was evaporated under reduced pressure to furnish the pure compounds.

\section{1-(3,5-Dimethyl-4-(phenyldiazenyl)-1H-pyrazol-1-yl)-2-((2-methylquinolin-8-} yl)oxy)ethanone (8a)

Recrystallised from ethanol as yellow crystals. Yield 81\%; m.p: $221-223{ }^{0} \mathrm{C}$; IR $\left(\mathrm{cm}^{-1}\right): 3050$ (CH stretching in aromatics), $2925\left(\mathrm{CH}\right.$ stretching in $\left.\mathrm{CH}_{3} / \mathrm{CH}_{2}\right), 1660(\mathrm{C}=\mathrm{O}$ amide), 1610 (C=N stretching), 1505, 1415 ( $\mathrm{N}=\mathrm{N}$ stretch), 1270, $1030\left(\mathrm{sp}^{2} / \mathrm{sp}^{3} \mathrm{C}-\mathrm{O}\right.$ stretching); ${ }^{1} \mathrm{H}$ NMR (DMSO-d $6,300 \mathrm{MHz}): \delta 2.26\left(\mathrm{~s}, 3 \mathrm{H}, \mathrm{CH}_{3}\right), 2.47\left(\mathrm{~s}, 3 \mathrm{H}, \mathrm{CH}_{3}\right), 2.66\left(\mathrm{~s}, 3 \mathrm{H}, \mathrm{CH}_{3}\right), 4.82(\mathrm{~s}, 2 \mathrm{H}$, $\left.\mathrm{OCH}_{2}\right), 7.26(\mathrm{~d}, 1 \mathrm{H}$, quinoline ring $\mathrm{H} 3), 8.34(\mathrm{~d}, 1 \mathrm{H}$, quinoline ring $\mathrm{H} 4), 7.58(\mathrm{~d}, 1 \mathrm{H}$, quinoline ring $\mathrm{H} 5), 7.54(\mathrm{t}, 1 \mathrm{H}$, quinoline ring $\mathrm{H} 6), 7.52(\mathrm{~d}, 1 \mathrm{H}$, quinoline ring $\mathrm{H} 7), 7.08-7.23(\mathrm{~m}, 5 \mathrm{H}$, Ar-H) ${ }^{13} \mathrm{C}$ NMR (DMSO-d 6 , $\left.300 \mathrm{MHz}\right): \delta 8.25\left(\mathrm{CH}_{3}\right.$ pyrazole ring), $14.6\left(\mathrm{CH}_{3}\right.$ pyrazole ring), $20.5\left(\mathrm{CH}_{3}\right.$ quinoline ring), 153.6, 122.2, 136.4, 121.7, 123.8, 111.7, 154.6, 139.6, 130.4 (quinoline ring), $73.4\left(\mathrm{OCH}_{2}\right), 163.2(\mathrm{C}=\mathrm{O}$ amide), 143.5, 108.3, 137.5 (pyrazole ring), 128.8, 129.3(2), 129.1(2), 129.3 (aromatic ring); Mass : $\mathrm{m} / \mathrm{z} 400.17[\mathrm{M}+\mathrm{H}]\left(\mathrm{M}^{+} 399.17\right)$. Anal. Calcd for $\mathrm{C}_{23} \mathrm{H}_{21} \mathrm{~N}_{5} \mathrm{O}_{2}$ : C, 69.16; H, 5.30; N, 17.53. Found: C, 68.96; H, 5.19; N, 17.41\%. 


\section{1-(3,5-Dimethyl-4-(p-tolyldiazenyl)-1H-pyrazol-1-yl)-2-((2-methylquinolin-8-yl)} oxy) ethanone $(\mathbf{8 b})$

Recrystallised from ethanol as gold crystals. Yield 79\%; m.p: 193-195 ${ }^{0} \mathrm{C}$. IR $\left(\mathrm{cm}^{-1}\right): 3060$ (CH stretching in aromatics), $2925\left(\mathrm{CH}\right.$ stretching in $\left.\mathrm{CH}_{3} / \mathrm{CH}_{2}\right), 1670(\mathrm{C}=\mathrm{O}$ stretching in amide), $1610(\mathrm{C}=\mathrm{N}$ stretching $), 1505(\mathrm{~N}=\mathrm{N}$ stretch $), 1270,1015\left(\mathrm{sp}^{2} / \mathrm{sp}^{3} \mathrm{C}-\mathrm{O}\right.$ stretching $) ;{ }^{1} \mathrm{H}$ NMR (DMSO-d $6,300 \mathrm{MHz}): \delta 2.23\left(\mathrm{~s}, 3 \mathrm{H}, \mathrm{CH}_{3}\right), 2.47\left(\mathrm{~s}, 3 \mathrm{H}, \mathrm{CH}_{3}\right), 2.35\left(\mathrm{~s}, 3 \mathrm{H}, \mathrm{CH}_{3}\right)$, $2.67\left(\mathrm{~s}, 3 \mathrm{H}, \mathrm{CH}_{3}\right), 4.85\left(\mathrm{~s}, 2 \mathrm{H}, \mathrm{OCH}_{2}\right), 7.26(\mathrm{~d}, 1 \mathrm{H}$, quinoline ring $\mathrm{H} 3), 8.32(\mathrm{~d}, 1 \mathrm{H}$, quinoline ring $\mathrm{H} 4), 7.61(\mathrm{~d}, 1 \mathrm{H}$, quinoline ring $\mathrm{H} 5), 7.52(\mathrm{t}, 1 \mathrm{H}$, quinoline ring $\mathrm{H} 6), 7.50(\mathrm{~d}$, $1 \mathrm{H}$, quinoline ring H7), $7.20(\mathrm{~d}, 2 \mathrm{H}, \mathrm{Ar}-\mathrm{H}), 7.85(\mathrm{~d}, 2 \mathrm{H}, \mathrm{Ar}-\mathrm{H}) ;{ }^{13} \mathrm{C}$ NMR (DMSO- $\mathrm{d}_{6}, 300$ $\mathrm{MHz})$ : $\delta 7.9\left(\mathrm{CH}_{3}\right.$ pyrazole ring $), 12.8\left(\mathrm{CH}_{3}\right.$ pyrazole ring $), 20.4\left(\mathrm{CH}_{3}\right.$ quinoline ring $), 23.7$ $\left(\mathrm{CH}_{3}\right.$ aromactic ring $), 154.4,122.3,135.9,121.9,123.5,112.4,155.3,139.1,129.5$ (quinoline ring), $72.2\left(\mathrm{OCH}_{2}\right), 162.6(\mathrm{C}=\mathrm{O}$ amide), 144.2, 107.6, 137.2 (pyrazole ring), 123.4, 127.5(2), 129.2(2), 136.7 (aromatic ring); Anal. Calcd for $\mathrm{C}_{24} \mathrm{H}_{23} \mathrm{~N}_{5} \mathrm{O}_{2}: \mathrm{C}, 69.72 ; \mathrm{H}$, $5.61 ; \mathrm{N}, 16.94$. Found: C, 69.61; H, 5.50; N, 16.79\%.

1-(4-((4-Chlorophenyl)diazenyl)-3,5-dimethyl-1H-pyrazol-1-yl)-2-((2methylquinolin-8-yl)oxy)ethanone $(8 \mathrm{c})$

Recrystallised from ethanol as dark red crystals. Yield $82 \%$; m.p: $224-226{ }^{0} \mathrm{C}$. IR $\left(\mathrm{cm}^{-1}\right)$ : $3060\left(\mathrm{CH}\right.$ stretching in aromatics), $2920\left(\mathrm{CH}\right.$ stretching in $\left.\mathrm{CH}_{3} / \mathrm{CH}_{2}\right), 1680(\mathrm{C}=\mathrm{O}$ stretching in amide $), 1615(\mathrm{C}=\mathrm{N}$ stretching $), 1510(\mathrm{~N}=\mathrm{N}$ stretch $), 1180,1080\left(\mathrm{sp}^{2} / \mathrm{sp}^{3} \mathrm{C}-\mathrm{O}\right.$ stretching $)$; ${ }^{1} \mathrm{H}$ NMR (DMSO-d $\left.6,300 \mathrm{MHz}\right): \delta 2.41\left(\mathrm{~s}, 3 \mathrm{H}, \mathrm{CH}_{3}\right), 2.52\left(\mathrm{~s}, 3 \mathrm{H}, \mathrm{CH}_{3}\right), 2.68\left(\mathrm{~s}, 3 \mathrm{H}_{1} \mathrm{CH}_{3}\right)$, $4.82\left(\mathrm{~s}, 2 \mathrm{H}, \mathrm{OCH}_{2}\right), 7.26(\mathrm{~d}, 1 \mathrm{H}$, quinoline ring $\mathrm{H} 3), 8.35(\mathrm{~d}, 1 \mathrm{H}$, quinoline ring $\mathrm{H} 4), 7.63(\mathrm{~d}$, $1 \mathrm{H}$, quinoline ring $\mathrm{H} 5), 7.58(\mathrm{t}, 1 \mathrm{H}$, quinoline ring $\mathrm{H} 6), 7.52(\mathrm{~d}, 1 \mathrm{H}$, quinoline ring $\mathrm{H} 7), 7.21$ (d, $2 \mathrm{H}, \mathrm{Ar}-\mathrm{H}), 7.25$ (d, 2H, Ar-H); ${ }^{13} \mathrm{C}$ NMR (DMSO-d 6 , $\left.300 \mathrm{MHz}\right): \delta 7.9\left(\mathrm{CH}_{3}\right.$ pyrazole ring), $13.6\left(\mathrm{CH}_{3}\right.$ pyrazole ring $), 20.5\left(\mathrm{CH}_{3}\right.$ quinoline ring ring $), 152.7,121.8,136.1,121.5,123.7$, 113.5, 156.2, 138.8, 128.8 (quinoline ring), $73.4\left(\mathrm{OCH}_{2}\right), 164.6(\mathrm{C}=\mathrm{O}$ amide), 142.5, 108.4, 136.7 (pyrazole ring), 123.7, 129.4(2), 128.2(2), 135.3 (aromatic ring); Anal. Calcd for $\mathrm{C}_{23} \mathrm{H}_{20} \mathrm{ClN}_{5} \mathrm{O}_{2}$ : C, 63.67; H, 4.65; N, 16.14. Found: C, 63.55; H, 4.54; N, 16.05.

1-(3,5-Dimethyl-4-((4-nitrophenyl)diazenyl)-1H-pyrazol-1-yl)-2-((2-methylquinolin8-yl)oxy)ethanone $(\mathbf{8 d})$

Recrystallised from ethanol as yellow crystals. Yield 75\%; m.p: 243-246 ${ }^{0} \mathrm{C}$. IR $\left(\mathrm{cm}^{-1}\right)$ : $3050\left(\mathrm{CH}\right.$ stretching in aromatics), $2925\left(\mathrm{CH}\right.$ stretching in $\left.\mathrm{CH}_{3} / \mathrm{CH}_{2}\right), 1680(\mathrm{C}=\mathrm{O}$ stretching in amide), 1615 ( $\mathrm{C}=\mathrm{N}$ stretching), 1515, $1405(\mathrm{~N}=\mathrm{N}$ stretch), 1365 (aromatic/heterocyclic ring vibrations), $1270,1080\left(\mathrm{sp}^{2} / \mathrm{sp}^{3} \mathrm{C}-\mathrm{O}\right.$ stretching); ${ }^{1} \mathrm{H}$ NMR (DMSO-d $\left.6,300 \mathrm{MHz}\right): \delta$ $2.17\left(\mathrm{~s}, 3 \mathrm{H}, \mathrm{CH}_{3}\right), 2.31\left(\mathrm{~s}, 3 \mathrm{H}, \mathrm{CH}_{3}\right), 2.59\left(\mathrm{~s}, 3 \mathrm{H}, \mathrm{CH}_{3}\right), 5.00\left(\mathrm{~s}, 2 \mathrm{H}, \mathrm{OCH}_{2}\right), 7.06(\mathrm{~d}, 2 \mathrm{H}$, quinoline ring $\mathrm{H} 3), 8.39(\mathrm{~d}, 1 \mathrm{H}$, quinoline ring $\mathrm{H} 4), 7.60(\mathrm{~d}, 1 \mathrm{H}$, quinoline ring $\mathrm{H} 5), 7.55(\mathrm{t}$, $1 \mathrm{H}$, quinoline ring $\mathrm{H} 6), 7.30(\mathrm{~d}, 2 \mathrm{H}$, quinoline ring $\mathrm{H} 7), 7.19(\mathrm{~d}, 2 \mathrm{H}, \mathrm{Ar}-\mathrm{H}), 8.27 \mathrm{~d}, 2 \mathrm{H}$, Ar-H); ${ }^{13} \mathrm{C}$ NMR (DMSO-d $\left.6,300 \mathrm{MHz}\right): \delta 8.2\left(\mathrm{CH}_{3}\right.$ pyrazole ring), $14.3\left(\mathrm{CH}_{3}\right.$ pyrazole ring), $20.5\left(\mathrm{CH}_{3}\right.$ quinoline ring $), 153.2,122.5,136.2,121.7,123.8,114.8,156.6,139.3$, 129.0 (quinoline ring), $73.5\left(\mathrm{OCH}_{2}\right), 164.8(\mathrm{C}=\mathrm{O}$ amide), 143.2, 110.3, 138.9 (pyrazole ring), 133.1, 123.2(2), 123.3(2), 151.9 (aromatic ring); Anal. Calcd for $\mathrm{C}_{23} \mathrm{H}_{20} \mathrm{~N}_{6} \mathrm{O}_{4}$ : C, 62.16; H, 4.54; N, 18.91. Found: C, 62.03; H, 4.45; N, $18.82 \%$.

1-(4-((2-Methoxyphenyl)diazenyl)-3,5-dimethyl-1H-pyrazol-1-yl)-2-((2methylquinolin-8-yl)oxy)ethanone (8e)

Recrystallised from ethanol as brown crystals. Yield 74\%; m.p: $185-187^{0} \mathrm{C}$. IR $\left(\mathrm{cm}^{-1}\right): 3030$ $\left(\mathrm{CH}\right.$ stretching in aromatics), $2920\left(\mathrm{CH}\right.$ stretching in $\left.\mathrm{CH}_{3} / \mathrm{CH}_{2}\right), 1680(\mathrm{C}=\mathrm{O}$ stretching 
in amide), $1615\left(\mathrm{C}=\mathrm{N}\right.$ stretching), $1510(\mathrm{~N}=\mathrm{N}$ stretch $), 1235,1025\left(\mathrm{sp}^{2} / \mathrm{sp}^{3} \mathrm{C}-\mathrm{O}\right.$ stretching); ${ }^{1} \mathrm{H}$ NMR (DMSO-d $\left.6,300 \mathrm{MHz}\right): \delta 2.46\left(\mathrm{~s}, 3 \mathrm{H}, \mathrm{CH}_{3}\right), 2.55\left(\mathrm{~s}, 3 \mathrm{H}, \mathrm{CH}_{3}\right), 2.69\left(\mathrm{~s}, 3 \mathrm{H}_{1} \mathrm{CH}_{3}\right)$, $3.92\left(\mathrm{~s}, 3 \mathrm{H}, \mathrm{OCH}_{3}\right), 4.85\left(\mathrm{~s}, 2 \mathrm{H}, \mathrm{OCH}_{2}\right), 7.21(\mathrm{~d}, 1 \mathrm{H}$, quinoline ring $\mathrm{H} 3), 8.32(\mathrm{~d}, 1 \mathrm{H}$, quinoline ring $\mathrm{H} 4), 7.60(\mathrm{t}, 1 \mathrm{H}$, quinoline ring $\mathrm{H} 5), 7.56(\mathrm{t}, 1 \mathrm{H}$, quinoline ring $\mathrm{H} 6), 7.52(\mathrm{~d}$, $1 \mathrm{H}$, quinoline ring H7), 6.48-7.12 (m, 4H, Ar-H); ${ }^{13} \mathrm{C}$ NMR (DMSO-d 6 , $\left.300 \mathrm{MHz}\right): \delta 7.9$ $\left(\mathrm{CH}_{3}\right.$ pyrazole ring), $13.1\left(\mathrm{CH}_{3}\right.$ pyrazole ring), $20.4\left(\mathrm{CH}_{3}\right.$ quinoline ring $), 56.9\left(\mathrm{OCH}_{3}\right.$ aromatic ring), 153.4, 121.6, 136.4, 121.1, 123.8, 112.9, 155.2, 138.9, 128.5 (quinoline ring), $73.5\left(\mathrm{OCH}_{2}\right), 165.4$ (C=O amide), 141.5, 108.3, 137.7 (pyrazole ring), 102.5, 158.6, 117.3, 123.2, 121.3, 131.6 (aromatic ring); Anal. Calcd for $\mathrm{C}_{24} \mathrm{H}_{23} \mathrm{~N}_{5} \mathrm{O}_{2}$ : C, 67.12; H, 5.40; N, 16.31. Found: C, 66.98; H, 5.35; N, 16.24\%.

\section{1-(4-((2-chlorophenyl)diazenyl)-3,5-dimethyl-1H-pyrazol-1-yl)-2-((2- methylquinolin-8-yl)oxy)ethanone $(8 f)$}

Recrystallised from ethanol as gold crystals. Yield $82 \%$; m.p: $215-218{ }^{0} \mathrm{C}$. IR $\left(\mathrm{cm}^{-1}\right): 3070$ $\left(\mathrm{CH}\right.$ stretching in aromatics), $2920\left(\mathrm{CH}\right.$ stretching in $\left.\mathrm{CH}_{3} / \mathrm{CH}_{2}\right), 1670(\mathrm{C}=\mathrm{O}$ stretching in amide), $1615(\mathrm{C}=\mathrm{N}$ stretching $), 1515(\mathrm{~N}=\mathrm{N}$ stretch $), 1250,1030\left(\mathrm{sp}^{2} / \mathrm{sp}^{3} \mathrm{C}-\mathrm{O}\right.$ stretching $)$; ${ }^{1} \mathrm{H}$ NMR (DMSO-d $\left.6,300 \mathrm{MHz}\right): \delta 2.42\left(\mathrm{~s}, 3 \mathrm{H}, \mathrm{CH}_{3}\right), 2.50\left(\mathrm{~s}, 3 \mathrm{H}, \mathrm{CH}_{3}\right), 2.52\left(\mathrm{~s}, 3 \mathrm{H}, \mathrm{CH}_{3}\right)$, $4.74\left(\mathrm{~s}, 2 \mathrm{H}, \mathrm{OCH}_{2}\right), 7.19(\mathrm{~d}, 1 \mathrm{H}$, quinoline ring $\mathrm{H} 3), 8.36(\mathrm{~d}, 1 \mathrm{H}$, quinoline ring $\mathrm{H} 4), 7.59$ $(\mathrm{d}, 1 \mathrm{H}$, quinoline ring $\mathrm{H} 5), 7.52(\mathrm{t}, 1 \mathrm{H}$, quinoline ring $\mathrm{H} 6), 7.43(\mathrm{~d}, 1 \mathrm{H}$, quinoline ring H7), 7.20-7.25 (m, 4H); ${ }^{13} \mathrm{C}$ NMR (DMSO-d 6 , $\left.300 \mathrm{MHz}\right): \delta 7.9\left(\mathrm{CH}_{3}\right.$ pyrazole ring), 13.7 $\left(\mathrm{CH}_{3}\right.$ pyrazole ring $), 20.5\left(\mathrm{CH}_{3}\right.$ quinoline ring $), 152.7,122.4,136.5,121.8,123.8,111.7$, 156.2, 139.5, 129.4 (quinoline ring), $72.8\left(\mathrm{OCH}_{2}\right), 164.7(\mathrm{C}=\mathrm{O}$ amide), 141.6, 109.4, 138.4 (pyrazole ring), 125.0, 135.8, 128.9, 131.3, 123.9, 131.6 (aromatic ring); Anal. Calcd for $\mathrm{C}_{23} \mathrm{H}_{20} \mathrm{ClN}_{5} \mathrm{O}_{2}: \mathrm{C}, 63.67 ; \mathrm{H}, 4.65 ; \mathrm{N}, 16.14$. Found: C, 63.54; H, 4.58; N, $16.02 \%$.

\section{1-(3,5-Dimethyl-4-((2-nitrophenyl)diazenyl)-1H-pyrazol-1-yl)-2-((2-methylquinolin-} 8-yl)oxy)ethanone ( $8 \mathrm{~g})$

Recrystallised from ethanol as orange crystals. Yield 80\%; m.p: $169-171{ }^{0} \mathrm{C}$. IR $\left(\mathrm{cm}^{-1}\right): 3070$ $\left(\mathrm{CH}\right.$ stretching in aromatics), $2940\left(\mathrm{CH}\right.$ stretching in $\left.\mathrm{CH}_{3} / \mathrm{CH}_{2}\right), 1680(\mathrm{C}=\mathrm{O}$ stretching in amide), 1620 (C=N stretching), 1550, 1415 ( N=N stretch), 1370, 1265, $1070\left(\mathrm{sp}^{2} / \mathrm{sp}^{3} \mathrm{C}-\mathrm{O}\right.$ stretching); ${ }^{1} \mathrm{H}$ NMR (DMSO-d 6 , $\left.300 \mathrm{MHz}\right): \delta 2.18\left(\mathrm{~s}, 3 \mathrm{H}, \mathrm{CH}_{3}\right), 2.35\left(\mathrm{~s}, 3 \mathrm{H}, \mathrm{CH}_{3}\right), 2.75$ (s, $\left.3 \mathrm{H}, \mathrm{CH}_{3}\right), 5.03\left(\mathrm{~s}, 2 \mathrm{H}, \mathrm{OCH}_{2}\right), 7.30(\mathrm{~d}, 1 \mathrm{H}$, quinoline ring $\mathrm{H} 3), 8.35(\mathrm{~d}, 1 \mathrm{H}$, quinoline ring $\mathrm{H} 4), 7.60(\mathrm{~d}, 1 \mathrm{H}$, quinoline ring $\mathrm{H} 5), 7.56(\mathrm{t}, 1 \mathrm{H}$, quinoline ring $\mathrm{H} 6), 7.48(\mathrm{~d}, 1 \mathrm{H}$, quinoline ring $\mathrm{H} 7), 7.45(\mathrm{~d}, 1 \mathrm{H}, \mathrm{Ar}-\mathrm{H}), 8.91(\mathrm{~d}, 1 \mathrm{H}, \mathrm{Ar}-\mathrm{H}), 8.03-8.18(\mathrm{~m}, 2 \mathrm{H}, \mathrm{Ar}-\mathrm{H}) ;{ }^{13} \mathrm{C} \mathrm{NMR}$ (DMSO-d $\left.{ }_{6}, 300 \mathrm{MHz}\right): \delta 8.06\left(\mathrm{CH}_{3}\right.$ pyrazole ring), $13.6\left(\mathrm{CH}_{3}\right.$ pyrazole ring), $20.6\left(\mathrm{CH}_{3}\right.$ quinoline ring), 153.5, 122.4, 136.3, 121.8, 123.8, 111.9, 155.6, 140.3, 129.7 (quinoline ring), $72.9\left(\mathrm{OCH}_{2}\right), 165.3(\mathrm{C}=\mathrm{O}$ amide), 145.1, 107.4, 139.2 (pyrazole ring), 122.9, 154.5, 123.4, 131.2, 1334.8, 131.4 (aromatic ring); Anal. Calcd for $\mathrm{C}_{23} \mathrm{H}_{20} \mathrm{~N}_{6} \mathrm{O}_{4}: \mathrm{C}, 62.16 ; \mathrm{H}, 4.54$; N, 18.91. Found: C, 62.05; H, 4.46; N, 18.76\%.

\section{General procedure for the synthesis of compounds $\mathbf{9 a - h}$}

A mixture of methyl 3-oxo-2-(phenyldiazenyl)butanoate (7a-h) $(0.01$ mole) and 2-((2methylquinolin-8-yl)oxy)acetohydrazide(3) $(0.01 \mathrm{~mole})$ in ethanol $(20 \mathrm{~mL})$ was heated under reflux for $8 \mathrm{~h}$ on a water bath. After completion of the reaction, ethanol was evaporated; the residue was dissolved in water, neutralized with $\mathrm{NaHCO}_{3}$ and extracted with ether. The ether solution was evaporated under reduced pressure to furnish the pure compound. 


\section{3-Methyl-1-(2-((2-methylquinolin-8-yl)oxy)acetyl)-4-(2-phenylhydrazono)-1H-} pyrazol-5(4H)-one (9a)

Recrystallised from ethanol as yellow crystals. Yield 85\%; m.p: $188-190{ }^{0} \mathrm{C}$. IR $\left(\mathrm{cm}^{-1}\right): 3450$ (br, NH stretching), 3070 ( $\mathrm{CH}$ stretching in aromatics), $2970\left(\mathrm{CH}\right.$ stretching in $\left.\mathrm{CH}_{3} / \mathrm{CH}_{2}\right)$, 1660, 1640 ( $\mathrm{C}=\mathrm{O}$ stretching in amide/heterocyclic ring), 1615 ( $\mathrm{C}=\mathrm{N}$ stretching), 1290, 1070 $\left(\mathrm{sp}^{2} / \mathrm{sp}^{3} \mathrm{C}-\mathrm{O}\right.$ stretching); ${ }^{1} \mathrm{H}$ NMR (DMSO-d 6 , $\left.300 \mathrm{MHz}\right): \delta 2.45\left(\mathrm{~s}, 3 \mathrm{H}, \mathrm{CH}_{3}\right), 2.51(\mathrm{~s}, 3 \mathrm{H}$, $\left.\mathrm{CH}_{3}\right), 5.07\left(\mathrm{~s}, 2 \mathrm{H}, \mathrm{OCH}_{2}\right), 7.12(\mathrm{~d}, 1 \mathrm{H}$, quinoline ring $\mathrm{H} 3), 8.39(\mathrm{~d}, 1 \mathrm{H}$, quinoline ring $\mathrm{H} 4)$, $7.62(\mathrm{~d}, 1 \mathrm{H}$, quinoline ring $\mathrm{H} 5), 7.60(\mathrm{t}, 1 \mathrm{H}$, quinoline ring $\mathrm{H} 6), 7.47(\mathrm{~d}, 1 \mathrm{H}$, quinoline ring H7), 7.09, 7.30- 7.41 (m, 5H, Ar-H), 11.63 (s, 1H, NH); ${ }^{13} \mathrm{C}$ NMR (DMSO-d 6 , $300 \mathrm{MHz}$ ): $\delta$ $12.3\left(\mathrm{CH}_{3}\right.$ pyrazolin-5-one ring), $20.3\left(\mathrm{CH}_{3}\right.$ quinoline ring), 153.7, 122.0, 136.0, 121.9, 123.8, 111.9, 154.9, 139.6, 129.0 (quinoline ring), $67.8\left(\mathrm{OCH}_{2}\right), 165.0(\mathrm{C}=\mathrm{O}), 149.4,126.7$, 164.7 (pyrazolin-5-one ring), 142.0, 115.0(2), 129.3(2), 121.0 (aromatic ring); Mass : $\mathrm{m} / \mathrm{z}$ $402.15[\mathrm{M}+\mathrm{H}]\left(\mathrm{M}^{+} 401.15\right)$. Anal.Calcd for $\mathrm{C}_{23} \mathrm{H}_{19} \mathrm{~N}_{5} \mathrm{O}_{3}$ : C, 65.83; H, 4.77; N, 17.45. Found: C, 65.42; H, 4.64; N, 17.28.

\section{4-(2-(4-Methoxyphenyl)hydrazono)-3-methyl-1-(2-((2-methylquinolin-8-yl)} oxy)acetyl)-1H-pyrazol-5(4H)-one (9b)

Recrystallised from ethanol as brown crystals. Yield 83\%; m.p: $174-176{ }^{0} \mathrm{C}$. IR $\left(\mathrm{cm}^{-1}\right): 3400$ ( $\mathrm{NH}$ stretching), $3030\left(\mathrm{CH}\right.$ stretching in aromatics), $2970\left(\mathrm{CH}\right.$ stretching in $\left.\mathrm{CH}_{3} / \mathrm{CH}_{2}\right)$, 1690, 1680 ( $\mathrm{C}=\mathrm{O}$ stretching in amide/heterocyclic ring), 1610 ( $\mathrm{C}=\mathrm{N}$ stretching), 1245, 1060 $\left(\mathrm{sp}^{2} / \mathrm{sp}^{3} \mathrm{C}-\mathrm{O}\right.$ stretching); ${ }^{1} \mathrm{H}$ NMR (DMSO-d $\left.6,300 \mathrm{MHz}\right): \delta 2.51\left(\mathrm{~s}, 3 \mathrm{H}, \mathrm{CH}_{3}\right), 2.69(\mathrm{~s}, 3 \mathrm{H}$, $\left.\mathrm{CH}_{3}\right), 3.93\left(\mathrm{~s}, 3 \mathrm{H}, \mathrm{OCH}_{3}\right), 4.89\left(\mathrm{~s}, 2 \mathrm{H}, \mathrm{OCH}_{2}\right), 7.14(\mathrm{~d}, 1 \mathrm{H}$, quinoline ring $\mathrm{H} 3), 8.36(\mathrm{~d}, 1 \mathrm{H}$, quinoline ring $\mathrm{H} 4), 7.64(\mathrm{~d}, 1 \mathrm{H}$, quinoline ring $\mathrm{H} 5), 7.62(\mathrm{t}, 1 \mathrm{H}$, quinoline ring $\mathrm{H} 6), 7.42(\mathrm{t}$, $1 \mathrm{H}$, quinoline ring H7), $7.37(\mathrm{~m}, 2 \mathrm{H}, \mathrm{Ar}-\mathrm{H}), 7.55(\mathrm{~m}, 2 \mathrm{H}, \mathrm{Ar}-\mathrm{H}), 10.56(\mathrm{~s}, 1 \mathrm{H}, \mathrm{NH}) ;{ }^{13} \mathrm{C}$ NMR (DMSO-d 6 , $300 \mathrm{MHz}): \delta 12.1\left(\mathrm{CH}_{3}\right.$ pyrazolin-5-one ring), $21.0\left(\mathrm{CH}_{3}\right.$ quinoline ring), $57.4\left(\mathrm{OCH}_{3}\right), 153.7,121.8,137.3,120.0,123.9,110.3,156.5,138.2,129.8$ (quinoline ring), $67.1\left(\mathrm{OCH}_{2}\right), 166.4(\mathrm{C}=\mathrm{O}), 150.4,126.1,163.9$ (pyrazolin-5-one ring), 123.6, 118.9(2), 117.4(2), 155.4 (aromatic ring); Anal. Calcd for $\mathrm{C}_{23} \mathrm{H}_{21} \mathrm{~N}_{5} \mathrm{O}_{4}: \mathrm{C}, 64.03 ; \mathrm{H}, 4.91 ; \mathrm{N}, 16.23$. Found: C, 63.89; H, 4.85; N, 16.12.

4-(2-(3-Methyl-1-(2-((2-methylquinolin-8-yl)oxy)acetyl)-5-oxo-1H-pyrazol-4(5H)ylidene)hydrazinyl)benzenesulfonamide (9c)

Recrystallised from ethanol as yellow crystals. Yield 83\%; m.p: 106-108 ${ }^{0} \mathrm{C}$. IR $\left(\mathrm{cm}^{-1}\right): 3370$ (NH stretching), 3065 (CH stretching in aromatics), $2950\left(\mathrm{CH}\right.$ stretching in $\left.\mathrm{CH}_{3} / \mathrm{CH}_{2}\right), 1670$, $1660(\mathrm{C}=\mathrm{O}$ stretching in amide/heterocyclic ring), $1615(\mathrm{C}=\mathrm{N}$ stretching $), 1380,1230,1040$ ( $\mathrm{sp}^{2} / \mathrm{sp}^{3} \mathrm{C}-\mathrm{O}$ stretching); ${ }^{1} \mathrm{H}$ NMR (DMSO-d $\left.6,300 \mathrm{MHz}\right): \delta 2.09$ (s, 2H, NH ), $2.49\left(\mathrm{~s}, 3 \mathrm{H}, \mathrm{CH}_{3}\right.$ ), $2.67\left(\mathrm{~s}, 3 \mathrm{H}, \mathrm{CH}_{3}\right), 4.91\left(\mathrm{~s}, 2 \mathrm{H}, \mathrm{OCH}_{2}\right), 7.15(\mathrm{~d}, 1 \mathrm{H}$, quinoline ring $\mathrm{H} 3), 8.38(\mathrm{~d}, 1 \mathrm{H}$, quinoline ring $\mathrm{H} 4), 7.66(\mathrm{~d}, 1 \mathrm{H}$, quinoline ring $\mathrm{H} 5), 7.63(\mathrm{t}, 1 \mathrm{H}$, quinoline ring $\mathrm{H} 6), 7.48(\mathrm{~d}, 1 \mathrm{H}$, quinoline ring H7), 7.12 (d, 2H,Ar-H), 7.56 (d, 2H,Ar-H), 10.52 (s, 1H, NH); ${ }^{13} \mathrm{C}$ NMR (DMSO-d 6,300 $\mathrm{MHz}): \delta 12.3\left(\mathrm{CH}_{3}\right.$ pyrazolin-5-one ring), $20.5\left(\mathrm{CH}_{3}\right.$ quinoline ring), 152.6, 122.3, 136.8, 121.3, $124.8,111.5,156.9,139.2,128.8$ (quinoline ring), $68.2\left(\mathrm{OCH}_{2}\right), 165.8(\mathrm{C}=\mathrm{O}), 148.6,127.3$, 164.2 (pyrazolin-5-one ring), 145.0, 118.1(2), 131.6(2), 132.8 (aromatic ring); Anal. Calcd for $\mathrm{C}_{22} \mathrm{H}_{20} \mathrm{~N}_{6} \mathrm{O}_{5} \mathrm{~S}: \mathrm{C}, 54.99 ; \mathrm{H}, 4.20 ; \mathrm{N}, 17.49$. Found: C, 54.85; H, 4.12; N, 17.40.

4-(2-(4-Chlorophenyl)hydrazono)-3-methyl-1-(2-((2-methylquinolin-8yl)oxy)acetyl)-1H-pyrazol-5(4H)-one (9d)

Recrystallised from ethanol as grey crystals. Yield $81 \%$; m.p: $165-167{ }^{0} \mathrm{C}$. IR $\left(\mathrm{cm}^{-1}\right): 3450$ ( $\mathrm{NH}$ stretching), $3070\left(\mathrm{CH}\right.$ stretching in aromatics), $2930\left(\mathrm{CH}\right.$ stretching in $\left.\mathrm{CH}_{3} / \mathrm{CH}_{2}\right)$, 
1690, $1630(\mathrm{C}=\mathrm{O}$ stretching in amide/heterocyclic ring), $1590(\mathrm{C}=\mathrm{N}$ stretch $), 1240,1080$ $\left(\mathrm{sp}^{2} / \mathrm{sp}^{3} \mathrm{C}-\mathrm{O}\right.$ stretching); ${ }^{1} \mathrm{H}$ NMR (DMSO-d 6 , $\left.300 \mathrm{MHz}\right): \delta 2.25\left(\mathrm{~s}, 3 \mathrm{H}, \mathrm{CH}_{3}\right), 2.73(\mathrm{~s}, 3 \mathrm{H}$, $\left.\mathrm{CH}_{3}\right), 4.91\left(\mathrm{~s}, 2 \mathrm{H}, \mathrm{OCH}_{2}\right), 7.15(\mathrm{~d}, 1 \mathrm{H}$, quinoline ring $\mathrm{H} 3), 8.36(\mathrm{~d}, 1 \mathrm{H}$, quinoline ring $\mathrm{H} 4)$, $7.68(\mathrm{~d}, 1 \mathrm{H}$, quinoline ring $\mathrm{H} 5), 7.65(\mathrm{t}, 1 \mathrm{H}$, quinoline ring $\mathrm{H} 6), 7.51(\mathrm{~d}, 1 \mathrm{H}$, quinoline ring H7), 7.20 (d, 2H, Ar-H) 8.40 (d, 2H, Ar-H), 10.57(s, 1H, NH); ${ }^{13} \mathrm{C}$ NMR (DMSO-d 6,300 $\mathrm{MHz}): \delta 12.4\left(\mathrm{CH}_{3}\right.$ pyrazolin-5-one ring), $20.6\left(\mathrm{CH}_{3}\right.$ quinoline ring), 153.2, 123.1, 136.3, 122.4 123.7, 111.8, 157.3, 139.2, 129.1 (quinoline ring), 67.1 $\left(\mathrm{OCH}_{2}\right), 165.2(\mathrm{C}=\mathrm{O}), 149.0$, 126.4, 163.6 (pyrazolin-5-one ring), 140.2, 122.9(2), 132.2(2), 125.5 (aromatic ring); Anal. Calcd for $\mathrm{C}_{22} \mathrm{H}_{18} \mathrm{ClN}_{5} \mathrm{O}_{3}$ : C, 60.62; H, 4.16; N, 16.07. Found: C, 60.45; H, 4.05; N, 15.95.

\section{3-Methyl-1-(2-((2-methylquinolin-8-yl)oxy)acetyl)-4-(2-(4-nitrophenyl) hydrazono)- 1H-pyrazol-5(4H)-one (9e)}

Recrystallised from ethanol as brown crystals. Yield 85\%; m.p: $204-207{ }^{0} \mathrm{C}$. IR $\left(\mathrm{cm}^{-1}\right): 3440$ ( $\mathrm{NH}$ stretching), $3065\left(\mathrm{CH}\right.$ stretching in aromatics), $2920\left(\mathrm{CH}\right.$ stretching in $\left.\mathrm{CH}_{3} / \mathrm{CH}_{2}\right)$, 1670,1660 ( $\mathrm{C}=\mathrm{O}$ stretching in amide/heterocyclic ring), 1615 ( $\mathrm{C}=\mathrm{N}$ stretching), 1380, 1330 (heterocyclic ring vibrations), $1245,1060\left(\mathrm{sp}^{2} / \mathrm{sp}^{3} \mathrm{C}-\mathrm{O}\right.$ stretching); ${ }^{1} \mathrm{H}$ NMR (DMSO-d 6 , 300 $\mathrm{MHz}): \delta 2.31\left(\mathrm{~s}, 3 \mathrm{H}, \mathrm{CH}_{3}\right), 2.71\left(\mathrm{~s}, 3 \mathrm{H}, \mathrm{CH}_{3}\right), 4.91\left(\mathrm{~s}, 2 \mathrm{H}, \mathrm{OCH}_{2}\right), 7.14(\mathrm{~d}, 1 \mathrm{H}$, quinoline ring $\mathrm{H} 3), 8.35(\mathrm{~d}, 1 \mathrm{H}$, quinoline ring $\mathrm{H} 4), 7.67(\mathrm{~d}, 1 \mathrm{H}$, quinoline ring $\mathrm{H} 5), 7.62(\mathrm{t}, 1 \mathrm{H}$, quinoline ring $\mathrm{H6}), 7.46(\mathrm{~d}, 1 \mathrm{H}$, quinoline ring $\mathrm{H} 7), 7.37(\mathrm{~d}, 2 \mathrm{H}, \mathrm{Ar}-\mathrm{H}), 8.02(\mathrm{~d}, 2 \mathrm{H}, \mathrm{Ar}-\mathrm{H})$, $10.58(\mathrm{~s}, 1 \mathrm{H}, \mathrm{NH}) ;{ }^{13} \mathrm{C}$ NMR (DMSO-d 6 , $\left.300 \mathrm{MHz}\right): \delta 12.2\left(\mathrm{CH}_{3}\right.$ pyrazolin-5-one ring), 21.2 $\left(\mathrm{CH}_{3}\right.$ quinoline ring), 148.6, 122.7, 136.1, 121.6, 123.8, 111.7, 156.2, 139.4, 129.1 (quinoline ring), $66.8\left(\mathrm{OCH}_{2}\right), 166.2(\mathrm{C}=\mathrm{O}), 147.5,127.8,163.8$ (pyrazolin-5-one ring), 148.7, 131.2(2), 123.3(2), 138.2 (aromatic ring); Anal. Calcd for $\mathrm{C}_{22} \mathrm{H}_{18} \mathrm{~N}_{6} \mathrm{O}_{5}: \mathrm{C}, 59.19 ; \mathrm{H}$, 4.06; N, 18.83. Found: C, 59.05; H, 3.89; N, 18.70.

\section{4-(2-(2-Methoxyphenyl)hydrazono)-3-methyl-1-(2-((2-methylquinolin-8-yl)oxy) acetyl)-1H-pyrazol-5(4H)-one (9f)}

Recrystallised from ethanol as orange crystals. Yield 80\%; m.p: $213-215{ }^{0} \mathrm{C}$. IR $\left(\mathrm{cm}^{-1}\right): 3390$ ( $\mathrm{NH}$ stretching), $3045\left(\mathrm{CH}\right.$ stretching in aromatics), $2870\left(\mathrm{CH}\right.$ stretching in $\left.\mathrm{CH}_{3} / \mathrm{CH}_{2}\right)$, 1680, 1660 ( $\mathrm{C}=\mathrm{O}$ stretching in amide/heterocyclic ring), 1615 ( $\mathrm{C}=\mathrm{N}$ stretching), 1240, 1020 $\left(\mathrm{sp}^{2} / \mathrm{sp}^{3} \mathrm{C}-\mathrm{O}\right.$ stretching); ${ }^{1} \mathrm{H}$ NMR (DMSO-d 6 , $\left.300 \mathrm{MHz}\right): \delta 2.50\left(\mathrm{~s}, 3 \mathrm{H}, \mathrm{CH}_{3}\right), 2.52(\mathrm{~s}, 3 \mathrm{H}$, $\left.\mathrm{CH}_{3}\right), 3.92\left(\mathrm{~s}, 3 \mathrm{H}, \mathrm{OCH}_{3}\right), 5.07\left(\mathrm{~s}, 2 \mathrm{H}, \mathrm{OCH}_{2}\right), 7.17$ (d, $1 \mathrm{H}$, quinoline ring $\left.\mathrm{H} 3\right), 8.39(\mathrm{~d}, 1 \mathrm{H}$, quinoline ring $\mathrm{H} 4), 7.66(\mathrm{~d}, 1 \mathrm{H}$, quinoline ring $\mathrm{H} 5), 7.58(\mathrm{t}, 1 \mathrm{H}$, quinoline ring $\mathrm{H} 6), 7.52(\mathrm{~d}$, $1 \mathrm{H}$, quinoline ring $\mathrm{H} 7), 7.03-7.14(\mathrm{~m}, 3 \mathrm{H}, \mathrm{Ar}-\mathrm{H}), 7.28-7.31(\mathrm{~m}, 1 \mathrm{H}, \mathrm{Ar}-\mathrm{H}), 10.58(\mathrm{~s}, 1 \mathrm{H}$, $\mathrm{NH}) ;{ }^{13} \mathrm{C}$ NMR (DMSO-d $\left.6,300 \mathrm{MHz}\right): \delta 12.3\left(\mathrm{CH}_{3}\right.$ pyrazolin-5-one ring), $20.6\left(\mathrm{CH}_{3}\right.$ quinoline ring), $57.2\left(\mathrm{OCH}_{3}\right), 152.4,122.7,136.3,121.8,123.4,109.2,155.3,138.2,128.9$ (quinoline ring), $67.9\left(\mathrm{OCH}_{2}\right), 166.4(\mathrm{C}=\mathrm{O}), 147.6,126.4,164.6$ (pyrazolin-5-one ring), 127.7, 146.1, 117.9, 120.5, 122.8, 119.4 (aromatic ring); Anal. Calcd for $\mathrm{C}_{23} \mathrm{H}_{21} \mathrm{~N}_{5} \mathrm{O}_{4}$ : C, 64.03; H, 4.91; N, 16.23. Found: C, 63.91; H, 4.78; N, 16.08.

\section{4-(2-(2-Chlorophenyl)hydrazono)-3-methyl-1-(2-((2-methylquinolin-8-yl)oxy) acetyl)-1H-pyrazol-5(4H)-one $(9 \mathrm{~g})$}

Recrystallised from ethanol as red crystals. Yield 83\%; m.p: $185-187{ }^{0} \mathrm{C}$. IR $\left(\mathrm{cm}^{-1}\right): 3430$ (NH stretching), $3060\left(\mathrm{CH}\right.$ stretching in aromatics), $2945\left(\mathrm{CH}\right.$ stretching in $\left.\mathrm{CH}_{3} / \mathrm{CH}_{2}\right)$, $1670,1630(\mathrm{C}=\mathrm{O}$ stretching in amide/heterocyclic ring $), 1610(\mathrm{C}=\mathrm{N}$ stretching $), 1270,1045$ $\left(\mathrm{sp}^{2} / \mathrm{sp}^{3} \mathrm{C}-\mathrm{O}\right.$ stretching); ${ }^{1} \mathrm{H}$ NMR (DMSO-d $\left.\mathrm{d}_{6}, 300 \mathrm{MHz}\right): \delta 2.14\left(\mathrm{~s}, 3 \mathrm{H}, \mathrm{CH}_{3}\right), 2.50(\mathrm{~s}, 3 \mathrm{H}$, $\left.\mathrm{CH}_{3}\right), 4.89\left(\mathrm{~s}, 2 \mathrm{H}, \mathrm{OCH}_{2}\right), 7.29(\mathrm{~d}, 1 \mathrm{H}$, quinoline ring $\mathrm{H} 3), 8.89(\mathrm{~d}, 1 \mathrm{H}$, quinoline ring $\mathrm{H} 4)$, $8.36(\mathrm{~d}, 1 \mathrm{H}$, quinoline ring $\mathrm{H} 5), 7.60(\mathrm{t}, 1 \mathrm{H}$, quinoline ring $\mathrm{H} 6), 7.57(\mathrm{~d}, 1 \mathrm{H}$, quinoline ring 
$\mathrm{H} 7), 6.97-7.02(\mathrm{~m}, 2 \mathrm{H}, \mathrm{Ar}-\mathrm{H}), 7.40-7.42(\mathrm{~m}, 2 \mathrm{H}, \mathrm{Ar}-\mathrm{H}), 10.59(\mathrm{~s}, 1 \mathrm{H}, \mathrm{NH}) ;{ }^{13} \mathrm{C} \mathrm{NMR}$ (DMSO-d $\left.\mathrm{d}_{6}, 300 \mathrm{MHz}\right): \delta 12.4\left(\mathrm{CH}_{3}\right.$ pyrazolin-5-one ring), $20.6\left(\mathrm{CH}_{3}\right.$ quinoline ring), 149.3, $122.4,134.7,121.5,124.3,112.3,154.7,140.4,129.3$ (quinoline ring), $67.6\left(\mathrm{OCH}_{2}\right), 165.6$ $(\mathrm{C}=\mathrm{O}), 148.1,127.2,163.6$ (pyrazolin-5-one ring), 145.6, 123.5, 129.6, 120.8, 126.4, 119.5 (aromatic ring); Anal. Calcd for $\mathrm{C}_{22} \mathrm{H}_{18} \mathrm{ClN}_{5} \mathrm{O}_{3}: \mathrm{C}, 60.62 ; \mathrm{H}, 4.16 ; \mathrm{N}, 16.07$. Found: $\mathrm{C}$, $59.96 ; \mathrm{H}, 4.03 ; \mathrm{N}, 15.92 \%$.

3-Methyl-1-(2-((2-methylquinolin-8-yl)oxy)acetyl)-4-(2-(2-nitrophenyl) hydrazono)-1Hpyrazol-5(4H)-one $(\mathbf{9 h})$

Recrystallised from ethanol as orange crystals. Yield 75\%; m.p: $234-236{ }^{0} \mathrm{C}$. IR $\left(\mathrm{cm}^{-1}\right): 3450$ ( $\mathrm{NH}$ stretching), $3070\left(\mathrm{CH}\right.$ stretching in aromatics), $2930\left(\mathrm{CH}\right.$ stretching in $\left.\mathrm{CH}_{3} / \mathrm{CH}_{2}\right)$, $1680,1630(\mathrm{C}=\mathrm{O}$ stretching in amide/heterocyclic ring $), 1610(\mathrm{C}=\mathrm{N}$ stretching $), 1470,1310$ (hetercyclic ring vibrations), 1275, $1090\left(\mathrm{sp}^{2} / \mathrm{sp}^{3} \mathrm{C}-\mathrm{O}\right.$ stretching); ${ }^{1} \mathrm{H}$ NMR (DMSO- $\mathrm{d}_{6}, 300$ $\mathrm{MHz}): \delta 2.53\left(\mathrm{~s}, 3 \mathrm{H}, \mathrm{CH}_{3}\right), 2.71\left(\mathrm{~s}, 3 \mathrm{H}, \mathrm{CH}_{3}\right), 4.91\left(\mathrm{~s}, 2 \mathrm{H}, \mathrm{OCH}_{2}\right), 7.17(\mathrm{~d}, 1 \mathrm{H}$, quinoline ring $\mathrm{H} 3), 8.36(\mathrm{~d}, 1 \mathrm{H}$, quinoline ring $\mathrm{H} 4), 7.65(\mathrm{~d}, 1 \mathrm{H}$, quinoline ring $\mathrm{H} 5), 7.63(\mathrm{t}, 1 \mathrm{H}$, quinoline ring H6), 7.49 (d, 1H, quinoline ring H7), $7.02(\mathrm{~d}, 1 \mathrm{H}, \mathrm{Ar}-\mathrm{H}), 7.43(\mathrm{t}, 1 \mathrm{H}, \mathrm{Ar}-\mathrm{H})$, 7.58 (t, 1H, Ar-H), 8.02 (d, 1H, Ar-H), 10.59 (s, 1H, NH); ${ }^{13} \mathrm{C}$ NMR (DMSO-d 6 , $\left.300 \mathrm{MHz}\right)$ : $\delta 12.43\left(\mathrm{CH}_{3}\right.$ pyrazolin-5-one ring), $20.4\left(\mathrm{CH}_{3}\right.$ quinoline ring), 154.6, 123.0, 136.2, 120.3, 125.1, 112.3, 156.2, 138.9, 129.5 (quinoline ring), $67.5\left(\mathrm{OCH}_{2}\right), 165.2(\mathrm{C}=\mathrm{O}), 149.0,126.4$, 164.5 (pyrazolin-5-one ring), 138.0, 137.4, 116.9, 121.4, 133.9, 122.2 (aromatic ring); Anal. Calcd for $\mathrm{C}_{22} \mathrm{H}_{18} \mathrm{~N}_{6} \mathrm{O}_{5}$ : C, 59.19; H, 4.06; N, 18.83. Found: C, 59.02; H, 3.94; N, 18.69\%.

\section{Results and Discussion}

The synthesised compounds 8a-g and 9a-h were prepared as depicted in Scheme 1. Initially, ethyl 2-((2-methylquinolin-8-yl)oxy)acetate (2) was prepared by agitating a mixture of 2-methylquinolin-8-ol (1) with ethyl chloroacetate in the presence of $\mathrm{K}_{2} \mathrm{CO}_{3}$ in DMF. Compound (2) was converted into corresponding acetohydrazide by heating with hydrazine hydrate to give 2-((2-methylquinolin-8-yl)oxy)acetohydrazide (3). The reaction of aryl diazonium chloride (5a-h) with acetylacetone and ethyl acetoacetate yielded the corresponding 3-(phenyldiazenyl)pentane-2,4-dione (6a-g) and ethyl 3-oxo-2-(2phenylhydrazono)butanoate (7a-h). Reaction of compound (3) with 6a-g in ethanol resulted in the formation of 1-(3,5-dimethyl-4-(substitutedphenyldiazenyl)-1H-pyrazol-1-yl)-2-((2methylquinolin-8-yl)oxy)ethanones (8a-g) in good yields. IR spectrum of $\mathbf{8 a}$ revealed a band at $1415 \mathrm{~cm}^{-1}$ due to $\mathrm{N}=\mathrm{N}$ group. The ${ }^{1} \mathrm{H}$ NMR spectrum of $\mathbf{8 a}$ showed two singlets at $\delta 2.26$, 2.47 indicating the presence of a pair of $\mathrm{CH}_{3}$ groups in the pyrazole ring. A singlet was also observed at $\delta 4.82$ due to $\mathrm{OCH}_{2}$ protons. The mass spectrum of $8 \mathbf{a}$ showed a peak at $\mathrm{m} / \mathrm{z}$ 400.17 corresponding to $[\mathrm{M}+\mathrm{H}]$ ion.

Reaction of compound (3) with 7a-h in ethanol resulted in the formation of 3-methyl-1(2-((2-methylquinolin-8-yl)oxy)acetyl)-4-(2-substitutedphenylhydrazono)-1H-pyrazol-5(4H)one $(\mathbf{9 a - h})$ in good yields. The spectral data of 9a-g confirmed that these compounds exist in hydrazono form. The spectral data of 9a revealed a strong absorption band at $1640 \mathrm{~cm}^{-1}$ due to $\mathrm{C}=\mathrm{O}$ group. Low frequency carbonyl band may be assigned to $\mathrm{C}=\mathrm{O}$ group in pyrazolin-5-one participating in intra-molecular hydrogen-bonding with $\mathrm{NH}$ group. The ${ }^{1} \mathrm{H}$ NMR spectrum of 9a, showed a singlet at $\delta 11.63$ due to the presence of hydrogen-bonded NH group. The mass spectrum of 9a showed a peak at $m / z, 402.15$ corresponding to $[\mathrm{M}+\mathrm{H}]$ ion.

Elemental analyses and spectral data of 8a-g and 9a-h are consistent with the assigned structures (c.f. Experimental section). The results of antimicrobial studies are summarized in Table 1. 

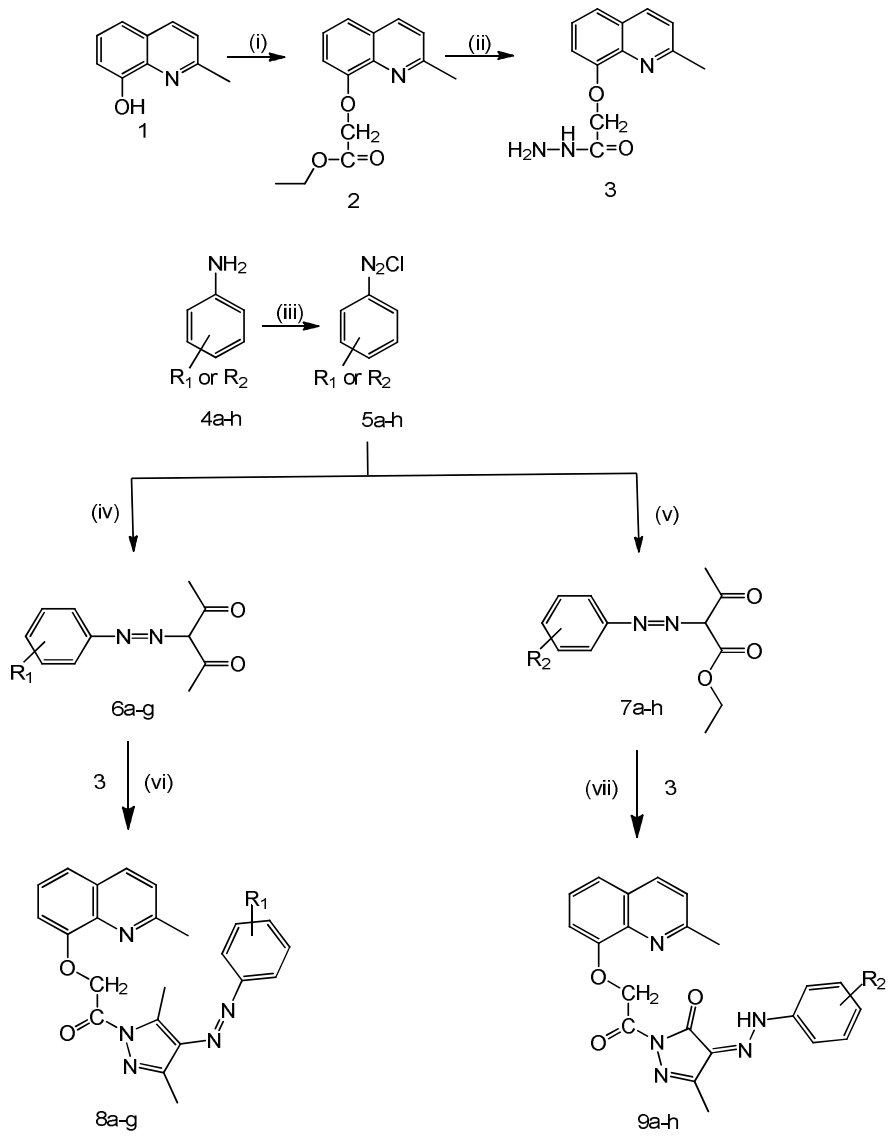

$\mathrm{R}_{1}=$ a) $-\mathrm{H}$ b) $4-\mathrm{CH}_{3}$ c) $4-\mathrm{Cl}$ d) $4-\mathrm{NO}_{2}$

$\mathrm{R}_{2}=$ a) $-\mathrm{H} \mathrm{b}$ ) $4-\mathrm{OCH}_{3}$ c) $4-\mathrm{SO}_{2} \mathrm{NH}_{2}$ d) $4-\mathrm{Cl}$

e) $2-\mathrm{OCH}_{3}$ f) $2-\mathrm{Cl} \mathrm{g}$ ) $2-\mathrm{NO}_{2}$

e) $4-\mathrm{NO}_{2}$ f) $2-\mathrm{OCH}_{3}$ g) 2-Cl h) $2-\mathrm{NO}_{2}$

(i) Ethyl chloroacetate $+\mathrm{K}^{2} \mathrm{CO}^{3} / \mathrm{DMF}$ (ii) $\mathrm{NH}^{2} \mathrm{NH}^{2} / \mathrm{C}^{2} \mathrm{H}^{6} \mathrm{OH}$ (iii) $\mathrm{NaNO}_{2} / \mathrm{HCl} /$ 0-5 ${ }^{\circ} \mathrm{C}$ (iv)

Acetylacetone / AcONa / EtOH (v) Ethyl actoacetate / AcONa / EtOH (vi) EIOH (vii) EtOH

\section{Scheme 1}

\section{Antimicrobial activity}

The antibacterial and antifungal activity of the synthesized compounds was examined by cup plate method against the following bacterial strains: Staphylococcus aureus, Bacillus subtilis, Escherichia coli, Pseudomonas aeruginosa, Salmonella typhi and fungi A. Niger, $U$. maydis, as compared to the standard drugs Gentamicin and Nystatin for bacterial and fungal growth respectively ${ }^{23,24}$.

The investigation of antibacterial screening data revealed that all the tested compounds showed moderate to good bacterial inhibition. But majority of the compounds did not exhibit any significant antifungal inhibition.

All the tested compounds were found to be potent against Gram-positive bacteria Staphylococcus aureus and Bacillus subtilis. Among them compounds 8a, 8b, 8d, 9d and 9f were found to be more potent against Staphylococcus aureus. Compounds $3,8 \mathbf{8}, \mathbf{8 b}, \mathbf{9 a}, \mathbf{9 b}$ and 9d were found to more potent against Bacillus subtilis. 
Table 1. In vitro antibacterial and antifungal activity

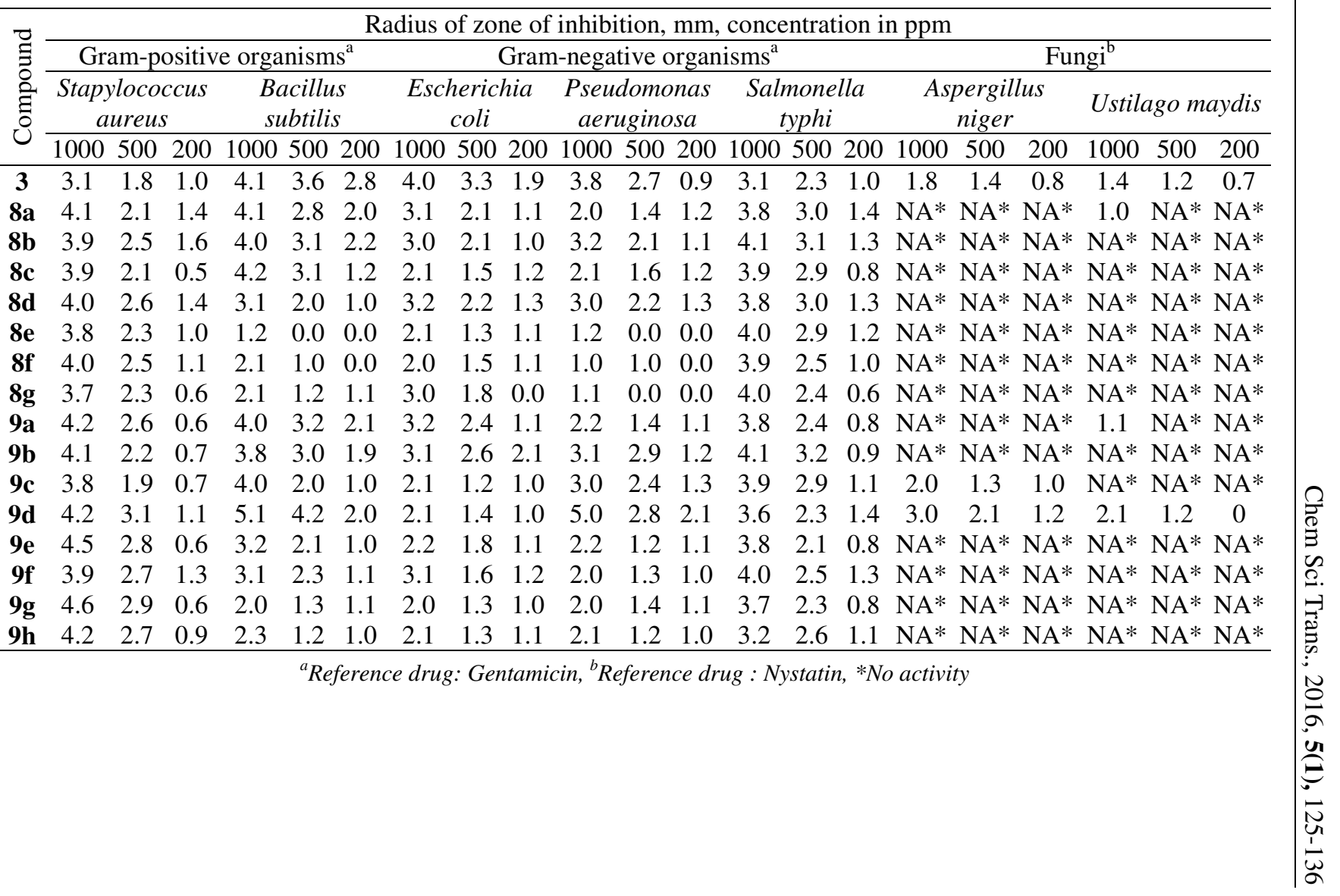


Similarly all tested compounds were found to be potent against Gram-negative bacteria Escherichia coli, Pseudomonas aeruginosa, Salmonella typhi. Compounds 3, 8d and 9b were found to be more potent against Escherichia coli. Compounds $8 \mathbf{d}$ and $9 \mathbf{d}$ were found to be more potent against Pseudomonas aeruginosa. Compounds $8 \mathbf{a}, 8 \mathbf{b}, 8 \mathbf{d}, 8 \mathbf{8}, 9 \mathbf{d}$ and $9 \mathrm{f}$ were found to be more potent against Salmonella typhi.

But majority of the compounds did not exhibit any significant antifungal activity against A. Niger and $U$. maydis. Only compounds 3, 9c and 9d showed feeble activity against $A$. Niger where as compounds $\mathbf{3 , 8 a}, 9 \mathbf{a}$ and $9 \mathbf{d}$ exhibited feeble activity against $U$. maydis.

\section{Conclusion}

The compounds 1-(3,5-dimethyl-4-(substitutedphenyldiazenyl)-1H-pyrazol-1-yl)-2-((2methylquinolin-8-yl)oxy)ethanones (8a-g) and 3-methyl-1-(2-((2-methylquinolin-8yl)oxy)acetyl)-4-(2-substitutedphenylhydrazono)-1H-pyrazol-5(4H)-ones (9a-h) were synthesized by combining 2-methylquinoline ring with pyrazole and pyrazolin-5-one scaffolds respectively. The spectral data are consistent with the structure of the newly synthesized compounds. The antimicrobial activity of the synthesized compounds was evaluated by disc diffusion method. The results revealed that all the compounds synthesized exhibited moderate to good antibacterial activity.

\section{Acknowledgment}

The authors wish to thank the University Grants Commission, New Delhi for award of Junior Research Fellowships to Mr. G. Bheemaraju, Mr. M. Mahesh and Mr. G. Manjunath under SAP and DST, New Delhi for its support under FIST.

\section{References}

1. Eicher T and Hauptmann S, The Chemistry of Heterocycles. Structure, Reactions, Syntheses and Applications; $2^{\text {nd }}$ Edition, Wiley-VCH: 2003.

2. Dahm R, Human Genetics, 2008, 122(6), 565-581; DOI:10.1007/s00439-007-0433-0

3. Watson J D and Crick F H C, Nature, 1953, 171(4356), 737-738; DOI:10.1038/171737a0

4. National Academy of Sciences. Institute of Medicine. Food and Nutrition Board., ed 1998. Chapter 9 Vitamin $B_{12}$. Dietary Reference Intakes for Thiamin, Riboflavin, Niacin, Vitamin $\mathrm{B}_{6}$, Folate, Vitamin $\mathrm{B}_{12}$, Pantothenic Acid, Biotin and Choline, Washington D C, National Academy Press, pp. 346.

5. Lee M K, Kim M J and Kim N J, Tetrahedron Lett., 2003, 44(35), 6737-6740; DOI:10.1016/S0040-4039(03)01648-4

6. Nagaraju V, Srinivasulu R, Doraswamy K and Ramana P V, J Indian Chem Soc., 2011, 88, 293-298.

7. Elena Ma, Francisco Y, Seltzman H H, Anne F, Mitchell G A, Rider S L, Pertwee R G, Stevenson L A and Thomas B F, J Med Chem., 2002, 45(13), 2708-2719; DOI:10.1021/jm010498v

8. Nakamura T, Sato M, Kakinuma H, Miyata N, Taniguchi K, Bando K, Koda A and Karneo K J Med Chem., 2003, 46(25), 5416-5427; DOI:10.1021/jm020557k

9. Fang B, Zhou C H and Rao C X, Eur J Med Chem., 2010, 45(9), 4388-4398; DOI:10.1016/j.ejmech.2010.06.012

10. Walker J K, Selness S R, Devraj R V, Hepperla M E, Naing W, Shieh H, Kunambail R, Yong S, Flynn D L, Benson A G, Messing D M, Dice T C, Kim T, Lindmark R J, Monohan J B and Portanova J, et al., Bioorg Med Chem Lett., 2010, 20(), 2634-2638; DOI:10.1016/j.bmcl.2010.02.047 
11. Bondock S, Fadaly W and Metwally M A, Eur J Med Chem., 2010, 45(9), 36923701; DOI:10.1016/j.ejmech.2010.05.018

12. Christodoulou M S, Liekens S, Kasiotis K M and Haroutounion S A, Bioorg Med Chem., 2010, 18(12), 4338-4350; DOI:10.1016/j.bmc.2010.04.076

13. Cottineau B, Toto P, Marot C, Pipaud A and Chemault J, Bioorg Med Chem Lett., 2002, 12(16), 2105-2108; DOI:10.1016/S0960-894X(02)00380-3

14. Abunado N M, Hassaneen H M, Kondile N G and Migdad O A, Molecules, 2008, 13(7), 1501-1517; DOI:10.3390/molecules13071501

15. Mullican D M, Wilson W M, Cannor T D, Kostlan R C and Dyer D R, J Med Chem., 1993, 36(8), 1090-1099; DOI:10.1021/jm00060a017

16. Acharya B N, Thavaselvam D and Kaushik M B, Med Chem Res., 2008, 17(8), 487494; DOI:10.1007/s00044-008-9092-5

17. Gilbert A M, Bursavich M G, Lombardi S, Georgiadis K E, Reifenberg E, Flannery C and Morris E A, Bioorg Med Chem Lett., 2008, 18(24), 6454-6457; DOI:10.1016/j.bmcl.2008.10.065

18. Upadhayaya R S, Vandavasi J K,Vasireddy N R, Sharma V, Dixit S S and Chattopadhyaya J, Bioorg Med Chem., 2009, 17(7), 2830-2841; DOI:10.1016/j.bmc.2009.02.026

19. Gholap A R, Toti K S, Shirazi F, Kumari R, Bhat M K, Deshpande M V and Srinivasan K V, Bioorg Med Chem., 2007, 15(21), 6705-6715; DOI:10.1016/j.bmc.2007.08.009

20. Rossiter S, Peron S J, Whitfield P J and Jones K, Bioorg Med Chem Lett., 2005, 15(21), 4806-4808; DOI:10.1016/j.bmcl.2005.07.044

21. Srimal R C, Gulati K, Nityanand S and Dhawan B N, Pharmacol Res.. 1990, 22(3), 319-329; DOI:10.1016/1043-6618(90)90729-W

22. Furniss B S, Hannaford A J, Smith P W G and Tatchell A R, Vogel's Text Book of Practical Organic Chemistry; $5^{\text {th }}$ Edition, Addison Wesley Longman: 1989.

23. Alekshun M N and Levy S B, Cell., 2007, 128(6), 1037-1050; DOI:10.1016/j.cell.2007.03.004

24. Sundriyal S, Sharma R K and Jain R, Curr Med Chem., 2006, 13(11), 1321-1335; DOI:10.2174/092986706776873023 